Zdravko Kravanja, Miloš Bogataj (Editors), Proceedings of the $26^{\text {th }}$ European Symposium on Computer Aided Process Engineering - ESCAPE 26

June 12th -15th, 2016, Portorož, Slovenia (C) 2016 Elsevier B.V. All rights reserved.

http://dx.doi.org/10.1016/B978-0-444-63428-3.50393-3

\title{
An Ontological Approach to Chemical Engineering Curriculum Development
}

\author{
Madeleine Bussemaker ${ }^{\mathrm{a}}$, Nikos Trokanas ${ }^{\mathrm{a}, \mathrm{b}}$, Franjo Cecelja ${ }^{\mathrm{a}}$ \\ ${ }^{a}$ Department of Chemical and Process Engineering, Surrey University, Guildford, GU2 \\ $7 X H$, United Kingdom \\ ${ }^{b}$ Centre for International Manufacturing, Institute for Manufacturing, Department of \\ Engineering, University of Cambridge, Cambridge, CB3 OFS, UK
}

\begin{abstract}
Continuous reflection and evolution of curricula in chemical engineering is beneficial for adaptation to evolving industry requirements, novel technologies and enhances student experience by being up to date and inclusive of effective teaching strategies. To this end it was necessary to develop a method to enable a holistic reflection on the curriculum and to examine the effect and potential areas of improvement and change. The curriculum was modelled using semantic knowledge modelling through the development of an Ontology, ChEEdO in the Protégé 3.5 environment. ChEEdo models topics within the domain of chemical engineering (Topics), modules taught in chemical engineering courses (Modules) and the learning outcomes of these modules (LearningOutcomes). The learning outcomes were related to the topics using verb properties from Bloom's taxonomy and using the context of each learning outcome. The functionality of semantic reasoning via the ontology was demonstrated with a case study based on curriculum development. The output of the modelling results demonstrated that the ontology could be successfully utilised for curriculum development and this is discussed in relation to practicality and future direction.
\end{abstract}

Keywords: knowledge modelling, curriculum development, ontology, chemical engineering education.

\section{Introduction: Knowledge Modelling in Education}

Knowledge modelling features in curriculum development historically in the form of ontologies, as well as concept maps. Conceptual curriculum mapping was used as a tool to develop and validate engineering curricula based on the program outcomes (Morsi et al., 2007). The benefits of conceptual maps for the curriculum were that they could be used to: facilitate validation, enable student and teacher conceptualisation of the course, and improve quality and alignment. Similarly, concept maps are used for curricula in school education, which encouraged alignment, integration and communication amongst teachers and are used currently in UK high school education (BBC, accessed 2015.; Koppang, 2004). Whilst concept mapping is a valid tool for knowledge modelling for curricula, the additional use of properties, restrictions and inferences in ontology engineering provides more scope to probe and investigate the curriculum structure. Within high school curricula in the UK, an ontology for the description of the terminology was developed and enables organisation of learning resources and content discovery (BBC, accessed 2015). Ontology engineering in higher education curricula has been used for various reasons such as managing complexity (Dexter and Davies, 2009), curriculum development (Cassel et al., 2008), improving resources (Gašević and Hatala, 2006), curriculum review (Ronchetti and Sant, 2007) and content sequencing 
(Ronchetti and Sant, 2007). Some capabilities of knowledge systems in the domain of curricula are: separation of foundation material from more complex material, validation of a program, assessment alignment and validation, change management, decision making tool, and relationship inferences. This project aims to demonstrate the viability of knowledge based modelling for the chemical engineering curriculum development and review using the curriculum for Chemical Engineering at the University of Surrey.

\section{Methodology: Development of ChEEdO}

\subsection{Topic conceptualisation and modelling}

The topic concepts related to chemical engineering were modelled based on three object properties: taxonomy property is $A$, mereology property isPartOf and functional property Uses, as previously applied in the development of a computing educational ontology. (Cassel et al., 2008) The topics were firstly arranged into groups and subgroups using parent topics as guidance. For example, some key parent topics related to chemical engineering were: Mathematics, ScientificFundamentals, Thermofluids, ReactionEngineering and Measurement. Then, each parent topic has subgroups, which are considered to be subsections of the parent topic and were related using the isA property e.g. Engineering has subgroups ChemicalEngineering, MechanicalEngineering, BioSystemsEngineering etc. Each of the subgroups of Engineering are linked to the Engineering group via the taxonomic is A property. The subgroups follow the same relationship laws, which are applied to the parent topic. In order to link a topic that was considered as pre-learning for another topic, the relationship ' $B$ Uses $C$ ' was defined to imply topic $\mathrm{C}$ should be learnt prior to topic $\mathrm{B}$. For example 'ReactionEngineering Uses Chemistry' which also implies that the subgroups of reaction engineering also relate to chemistry with the verb Uses (Figure 1). Then, subgroups of ReactionEngineering may be related to subgroups of Chemistry. For example the subgroup ReactorKinetics uses theory covered in ChemicalReaction and ChemicalReactionEquation.

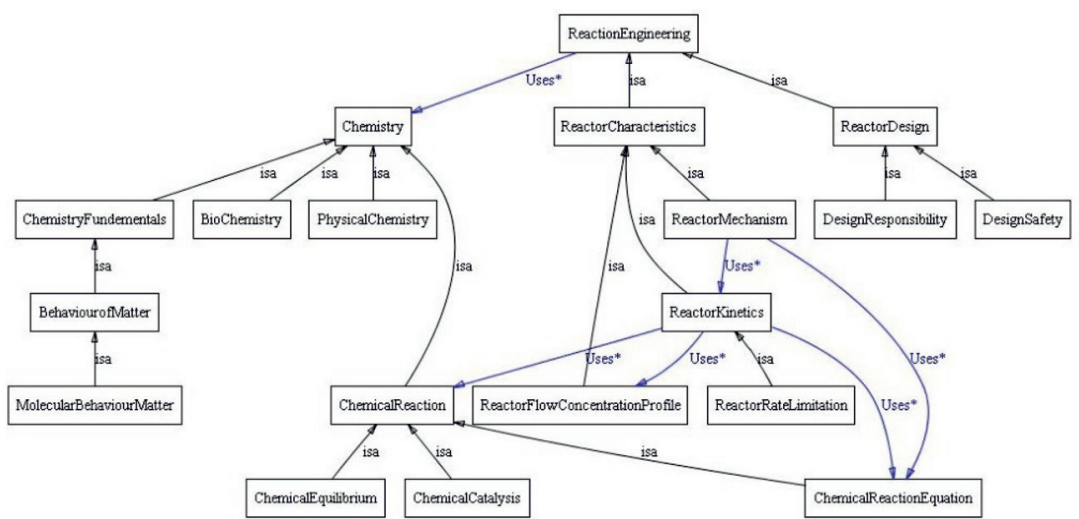

Figure 1. An example of the classification of topics and how the 'Uses' verb is applied.

The object property ' $A$ isPartOf $B$ ' implies that topic $A$ is a subsection of topic $B$ and topic A contributes toward the learning of topic B. An example of this is presented in Figure 2, where the mereology of ProcessAnalysis and ReactionEngineering are shown, and the transitive object property isPartOf demonstrated. The transitive nature of the property means that, if ProcessAnalysis isPartOf ReactionEngineering and 
ReactionEngineering isPartOf ProcessPlant then this implies that, ProcessAnalysis isPartOf ProcessPlant. The two other properties in the topic tree, isA, and Uses are also transitive. The demonstration model was based on the reaction engineering branch of chemical engineering, plus the first year curriculum. Each topic, and learning outcome is governed by a set of restrictions, which allows for semantic reasoning.

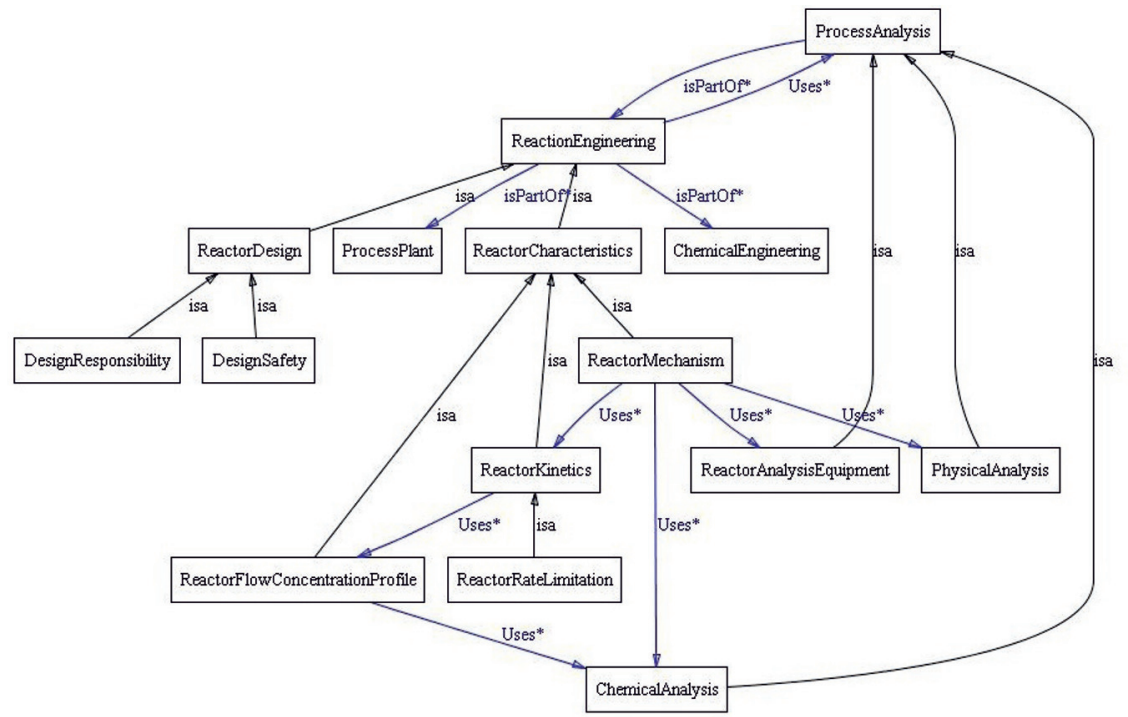

Figure 2. A representation of the two parent topics, ReactionEngineering and ProcessAnalysis.

\subsection{Learning outcome taxonomy and modelling}

In order to add meaning to the ontology, the learning outcomes were mapped according to the topic mereology. Recently, (2014-15) the module descriptors were modified so that the learning outcomes used Bloom's taxonomy (Bloom, 1956) and followed the structure as defined by Biggs. (Biggs and Tang, 2011) This meant that each learning outcome has a learning verb that defines the learning level reflected in the six learning levels defined by Bloom. The learning verb relates to a learning object and context, which defines the scope and topic of learning. In the semantic model the learning outcome was linked to the context via the property hasContext, then it was linked to the learning object via a learning verb from Bloom, under the superproperty hasLearningOf. The superproperty hasLearning $O f$ thus had five subproperties based upon the levels of learning defined in Bloom's taxonomy. The learning verb properties had inverse properties, and both the verb and inverse, were transitive so that reasoning could be performed. A list of the learning verbs and their inverse are given in Table 1.

Table 1. Properties used to describe learning outcomes and the level of learning

\begin{tabular}{c|cc}
\hline Learning Level & Learning Verb & Inverse Learning Verb \\
\hline & hasLearningOf (Parent verb) & isLearntIn (Parent inverse verb) \\
Knowledge (K) & hasKnowledgeOf & isKnownIn \\
Comprehension (Co) & hasComprehensionOf & isComprehendedln \\
Application (Ap) & hasApplicationOf & isAppliedIn \\
Analysis (An) & hasAnalysisOf & isAnalysedln \\
Evaluation (Ev) & hasEvaluationOf & isEvaluatedIn \\
Synthesis (S) & hasSynthesisOf & isSynthesisedln \\
\hline
\end{tabular}


The object and the context in the learning outcomes were found in the topic mereology. Thus, each learning outcome hasLearningOf TopicX and the learning outcome hasContext TopicY. An example of how this was constructed is given in Figure 3. In this figure, two learning outcomes are featured from a first year module, Scientific Fundamentals (SCFU). The construction of the semantic model begins with the learning outcomes as described in the module descriptor, and the identification of the key learning verb, learning object and context. Each learning outcome is linked to a module, which in turn belongs to a year level. The learning verbs are classified into one of the learning levels as listed in Table 1. The learning object and learning context are taken from the learning outcome statement as shown in Table 2. In some cases the context of the learning outcome is not clear and requires some inference or additional knowledge of the subject. This information is normally found within the module aims on the module descriptor, if not already known.

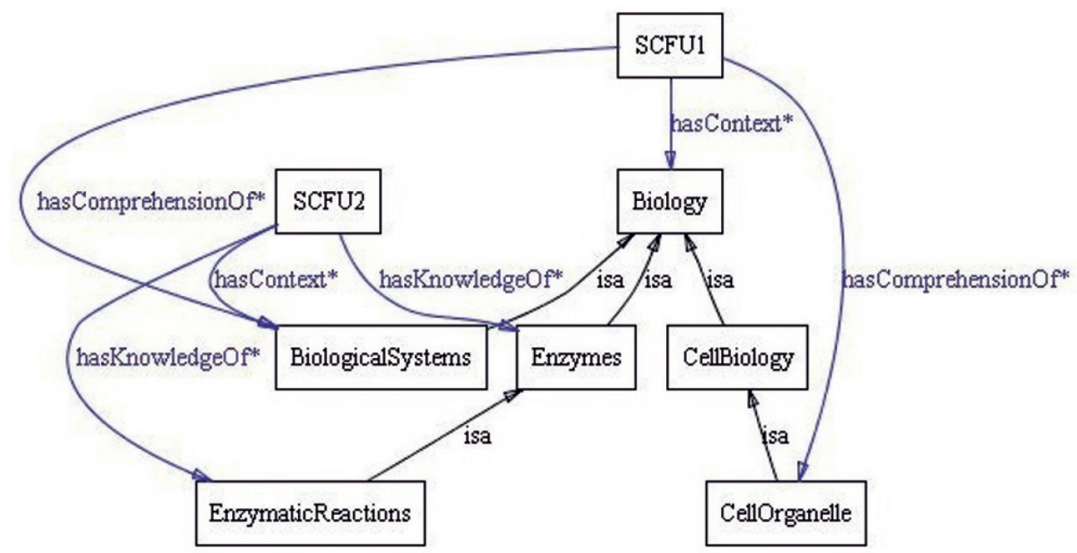

Figure 3. An example of mapping of two learning outcomes, SCFU1 and SCFU2, each with context and learning objects.

Table 2. Construction of the semantic model based on learning outcomes

\begin{tabular}{lllll}
\hline $\begin{array}{l}\text { 1080 Scientific Fundamentals: } \\
\text { Learning outcomes }\end{array}$ & Learning Verb & $\begin{array}{l}\text { Learning } \\
\text { Object }\end{array}$ & Context & Code \\
\hline $\begin{array}{llll}\text { Distinguish between the } \\
\text { function of different biological } \\
\text { systems and cell organelles. }\end{array}$ & Distinguish (Co) & $\begin{array}{l}\text {-Cell } \\
\text { organelle } \\
\text {-Biological } \\
\text { systems }\end{array}$ & Biology & SCFU1 \\
$\begin{array}{llll}\text { Describe and classify enzymes } \\
\text { and enzymatic reactions. }\end{array}$ & Describe (K) & Enzymes & $\begin{array}{l}\text { Biological } \\
\text { systems }\end{array}$ & SCFU2 \\
\hline
\end{tabular}

\section{Results and Discussion}

\subsection{Placing Learning Topics into Context}

Often students are displeased with some areas of fundamental sciences and do not see their use in later years. The topic description within the ontology is able to demonstrate which other areas of chemical engineering relate to fundamental learning topics. This can also aid in the identification of core and non-core areas of the curriculum. For 
example, if the query of which concept Uses the topic of Differentiation the results will include all of the concepts in the topic tree, that were defined to be using differentiation. Figure 4 shows the results of two queries, in relation to which topic Uses Sustainability and which topic Uses Differentiation. Here, the topic of ReactorDesign and IndustrialChemicalRegulations are returned as topics which UsesSustainability. This information can be used to demonstrate relevance of a topic to a student and to inform the curriculum developers about links within the learning pathway of the student.

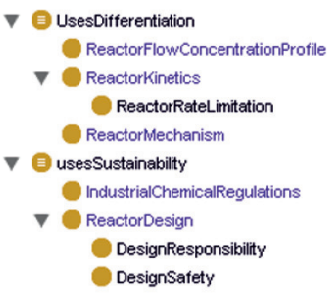

Figure 4. Results from two queries about which topic Uses Differentiation and Sustainability.

\subsection{Developing New Material in Context of Prior Learning}

Chemical engineering graduates can now be found in highly specialist areas such as molecular engineering, nanotechnology and microelectronics. (Byrne, 2006) To further develop the curriculum, new academics may want to introduce concepts at higher levels within the degree program or add specialisation. Specialisation options are often geospecific and may be reflected in the expertise and research interests of the staff teaching the degree. (Gomes et al., 2006) In terms of teaching efficacy it is often best to align teachers with fields of expertise in order to maintain enthusiasm and interest. However, when additional areas of interest are considered, the curriculum needs to be evaluated to consider what learning has been done in certain contexts in previous years.

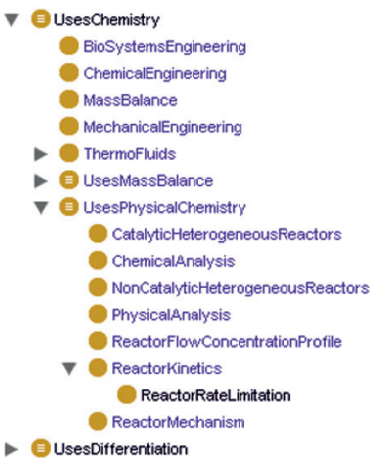

Figure 5. An additional query into topics which Uses PhysicalChemistry

For example we consider the development of additional learning about chemical and physical analysis at a third year level. It is known that the learning of these additional concepts requires knowledge of MolecularPhysicalChemisty in some context. Hence the specific topic of MolecularPhysicalChemisty is added to the topic taxonomy, underneath the group PhysicalChemistry. Then, it can be seen where PhysicalChemistry is used in other topics, as shown in Figure 5. The learning of these topics can be discovered using the ontology interface and information about the level of learning and context is easily 
extracted. Therefore, a decision about which context to place the additional learning material on physical and chemical analysis can be facilitated. This can be applied when developing new curriculum at higher levels where prior learning relevant to the new material needs to be assessed.

\section{Conclusions}

Here, a method to model a curriculum using the Ontology developer Protégé 3.5 was presented. The semantic model was created using links from educational concepts extracted from the learning outcomes as featured on the module descriptors for the chemical engineering degree program. The modelling used semantic reasoning in order to provide information and advice relating to curriculum structure and development. Through queries about the information in the ontology, core topics and learning relationships can be identified in order to assess curriculum development options.

\section{References}

BBC, Curriculum Ontology [WWW Document]. BBC Ontol. (Accessed 2015)

J. B. Biggs, C. S. Tang, 2011, Teaching for quality learning at university: what the student does. Open University Press, Maidenhead.

B. S. Bloom, 1956. Taxonomy of Educational Objectives: The Classification of Education Goals. Cognitive Domain. Handbook 1. Longman.

E. P. Byrne, 2006. The role of specialization in the chemical engineering curriculum. Educ. Chem. Eng. 1, 3-15.

L. N. Cassel, G. Davies, R. LeBlanc, L. Snyder, H. Topi, 2008. Using a computing ontology as a foundation for curriculum development, in: Proceedings of the Sixth International Workshop on Ontologies \& Semantic Web for E-Learning. 21-29.

H. Dexter, I. Davies, 2009. An ontology-based curriculum knowledgebase for managing complexity and change, in: Advanced Learning Technologies, 2009. ICALT 2009. Ninth IEEE International Conference on. IEEE, 136-140.

D. Gašević, M. Hatala, 2006. Ontology mappings to improve learning resource search. Br. J. Educ. Technol. 37, 375-389.

V. G. Gomes, G. W. Barton, J. G. Petrie, J. Romagnoli, P. Holt, A. Abbas, B. Cohen, A. T. Harris, B. S. Haynes, T. A. G. Langrish, 2006. Chemical engineering curriculum renewal. Educ. Chem. Eng. 1, 116-125.

IChemE (Ed.), 2011. Accreditation of chemical engineering degrees. www.icheme.org .

A. Koppang, 2004. Curriculum Mapping Building Collaboration and Communication. Interv. Sch. Clin. 39, 154-161.

R. Morsi, W. Ibrahim, F. Williams, 2007. Concept maps: Development and validation of engineering curricula, in: Frontiers In Education Conference-Global Engineering: Knowledge Without Borders, Opportunities Without Passports, 2007. FIE'07. 37th Annual. IEEE, pp. T3H-18-T3H-23.

M. Ronchetti, J. Sant, 2007. Curriculum management and review: an ontology-based solution, in: Proceedings of World Conference on E-Learning in Corporate, Government, Healthcare, and Higher Education, 2007, 6476-6482 